$\mathbf{A}$

\title{
CASE OF CESAREAN SECTION.
}

\author{
BY \\ DR. OLDHAM, \\ OBSTETRIC PHYSICIAN AND LECTURER ON MIDWIFERY, ETC., \\ AT GUY'S HOSPITAL.
}

Received January 14th.-Read February 11th, 1851.

SARAH -, æt. 23, engaged at waistcoat-making in Bethnal Green, was born a feeble child, and during childhood was weak and rickety. The mother states that she was 7 years old before she could put her feet to the ground, and 13 years of age before she could walk securely. At 16 she first began to menstruate, and has continued to do so with tolerable regularity ever since, and her general health has been fairly good. She is now four feet eight inches in height, walks lame, and her legs are curved, so that she retains a deformed and dwarfish appearance. Her face and features are small, and her countenance is that of a girl of 14 or 15 years of age, and her circulation habitually rapid.

She applied to me for advice in the middle of September, 1850, complaining of amenorrhœe, of seven months' standing, the result, as she declared, of cold, for which she had ineffectually taken various medicines. At this time the abdomen was prominently large, and by auscultation the beats of the fœtal heart and uterine murmur were readily detected. She denied, at this time, the possibility of pregnancy, but subsequently she admitted that she had had sexual intercourse on two occasions, but she was in so much alarm at the time that she thought it impossible that she could have become pregnant. The lower lumbar vertebræ and the sacrum were observed to be deeply curved, and the 
uterus was anteverted in a marked degree. On examination, the orifice of the vagina was found to retain much of the virgin contraction, from an imperfect laceration of the hymen; the canal itself was narrow and very tender, so that she shrunk from the necessary exploration. The projection of the promontory of the sacrum was so great as to shorten the conjugate diameter to about two inches; but the pelvic cavity and outlet were comparatively roomy. She was advised without loss of time to obtain a room near Guy's Hospital, and to be entered as a patient of the Lying-in Charity.

Sept. 23, 1850. This morning I determined to induce labour by puncturing the membranes, the bowels having previously been relieved by castor-oil. For this purpose a long slightly-curved canula, with a sharp-pointed stilette, which could be withdrawn at will from the canula, was employed. Some difficulty was experienced in reaching the os-uteri, as the patient lay on her side, on account of the height at which it was placed, the extreme tenderness of her soft structures, and her inability to flex her thighs well, which was caused by the prominence of the anteverted uterus. She was therefore placed on her back, and the canula having entered the amnial sac, about eight ounces of clear liquor amnii were drawn off. At this time the fœtal head could be felt, distinctly marked out on the abdomen above the pubes.

24th. This morning the bowels had been again relieved by aperient medicine, and some uneasiness about the uterus had been felt. Mr. Davies, who had charge of the case, examined and found the left arm in the vagina. On visiting her, I found that the child was still alive, and that touching the palm of the hand in the vagina, excited reflex muscular movement. The os uteri surrounded the arm, and the uterus was quiescent. The patient was ordered to keep quiet, and it was requested that no further vaginal examination should be made.

25th. She had not slept well on account of some irregular diffused uterine pain. The arm and os uteri were in the same 
condition. She had passed urine freely, and during the day was tolerably free from pain. Her pulse is rapid, 93 in the minute, which is natural to her; but there are no febrile symptoms, and she is in good spirits.

26th. Early this morning 3vj of castor-oil were given, which acted freely on the bowels in the course of the morning. At 9 a.m., some regular uterine pains commenced; and when I saw her the os uteri was found to be dilating well, and the vagina softer and less tender. The skin of the fotal arm was peeling off.

Vaginal examination was forbidden.

At half-past 9 p.m., the os uteri was fully dilated, but the loose soft margin of it could still be felt, on account of the fotal head being so held above the brim as not to have any bearing upon it. Some efforts at delivery were now made, the rectum and bladder being both empty.

A full exploration of the pelvic brim and the presenting part was made by the cautious introduction of the fingers of the left hand, with a view, if practicable, of running them beyond the brim, and catching and bringing down a foot; the right hand at the same time pressing the uterus from the abdomen, and doubling down towards the pelvic inlet the lower limbs of the foetus, in a direction to meet the hand in the vagina. The patient was at this time on her back, but the pelvic brim was so contracted that it was soon obvious that any hope of delivery in this way must be abandoned. Traction was then made on the protruding arm, which shortly gave way at the elbow-joint; but the efforts of traction were found to have some effect upon the head, in moving it from above the pubes to the brim, which was aided by external pressure, directing it from the one part to the other. After the arm had separated, the head was so far within reach that the perforator could be made to touch it, and it was opened without difficulty. After the brain had been emptied, and,under the influence of regular and powerful uterine action, the bones of the head had shrunk together, repeated efforts were made with the crotchet, applied within the skull and outside of it, to draw the head through the 
brim. In doing this the cranial bones and a part of the base of the skull were torn up, but without making any material way through the contracted brim. In the course of this operation, which was occasionally suspended for ten minutes to rest and refresh the patient, the right side of the pelvis was found to have squeezed into it the right hand and foot, both of which could be distinctly felt in this situation. A hope was now entertained that one or other of these limbs would double deeper into the pelvis, so as to be caught and drawn down, and attempts were made to accomplish this, but ineffectually. No hold could be got of either the foot or hand beyond the slippery tips of each, and labour-pains did not press them lower. A polypus canula and ligature were sent for, but the noose could not be passed over the ankle or wrist, and consequently there was no purchase for it. With the narrow brim of the pelvis thus packed by this complex presentation, the different parts of which mutually obstructed the process of each other's descent, and judging from the difficulties already experienced on attempting delivery-of the far greater ones which must be encountered in order to complete it-it became a question whether delivery should not be effected by the Cæsarean section. After a consideration of the case, I determined in favour of it, but before undertaking it, a consultation was held with my colleague, Dr. Lever, who having examined the patient, acquiesced in this decision, and without further delay preparations were made for performing it.

The room was heated by a good fire until the temperature was sensibly warm, some candles were obtained from the Hospital, and the Assistant-surgeon of the Hospital, Mr. Poland who operated, with the Resident Obstetric Clerks, soon arrived. The bladder was emptied by a catheter. The patient was placed completely under the influence of chloroform, and one gentleman had charge of the chloroform, and kept her during the operation under its power. The patient was drawn to the right side of the bed, the shoulders were slightly raised, and the best arrangements were made to let her maintain, without moving, the same position after the 
operation. One assistant was directed to close the abdomen as the uterus contracted on being emptied, and the act of delivery was undertaken by myself. An incision beginning about two inches above the umbilicus, and carried down to five inches below it, so as nearly to reach the pubes, was made in the course of the linea alba, just missing the umbilicus itself. No bleeding of consequence followed this incision, and the uterus was soon exposed. On opening the abdominal cavity, some three or four ounces of transparent straw-coloured fluid ran out, and were caught by a sponge. The body of the uterus was seen to contract in a perfectly even way, on thus being laid bare, and to form a hardened compact body, its surface having its natural polish, and neither the large veins nor smaller capillaries being strikingly injected. The omentum glided easily between the fundus uteri and the abdominal wall, with a descent of about two inches. The uterine wall was then cut through, and the direction of the incision was slightly curved, the convexity looking to the right side. The length of the opening was about five inches, and the incision was made to reach the lowest part of the uterus near the cervix, so as to open the womb from below. But little blood was lost. I then passed my right hand into the cavity, and withdrew a full sized seven months' fotus, by laying hold of its thigh. The uterus immediately contracted, and the intestines appeared at the upper part of the abdominal opening, which was promptly closed. The hand was again introduced between the membranes and the surface of the uterus, and with but little delay the placenta and membranes were removed, the former being attached to the upper part of the posterior wall. The uterus then contracted, and in doing so the convex border of the incised wall was seen to wrinkle, and not to adapt itself to the corresponding surface. Some venous bleeding took place, but to no great amount. A sponge wrung out of warm water was now gently drawn over that portion of the convex border of the intestines and omentum which had protruded and been smeared with blood, and the upper part of the external opening was at once closed by the application of sutures. Whilst this was 
being done by Mr. Poland, I held a sponge over the edges of the uterine opening, gently pressing them together, and by the time that the wound was closed above, all bleeding had ceased, and the two surfaces were evenly adapted, and were seen to be quite free from any interposed structure. Other sutures, eleven in all, were then applied; some broad strips of plaster were drawn across the abdomen, and two cushions of lint were placed vertically on the sides of the central incision, and a binder cut out into tails was adjusted over the whole. During the operation the patient was quiet, excepting when the uterus was at its height of contraction, when she wriggled about slightly, but hardly enough to incommode Mr. Poland. The pulse was of good strength, and she was left reclining on her back, and sleeping in a tranquil manner under the influence of chloroform.

At a quarter to four, a.m., three quarters of an hour after the operation, she opened her eyes as though waking from a natural sleep, and she then told me that she had known nothing of the operation, and felt quite comfortable. Just before this her pulse was 118 in the minute, and the respiration 32.

During the remainder of the night she took $z^{\mathrm{ij}}$ of laudanum ; at first, $3 j$ was given her soon after she awoke, half an hour after which she began to shiver; the pulse became rapid and weak; the respiration quickened and catching; the extremities cold; and she complained urgently of an oppressive tightness over the upper part of the abdomen. A small quantity of hot brandy-and-water was given her, and 3 ss of laudanum, and some warm clothes were tucked around her. The bandage was loosened at its upper part, which gave her some relief. In an hour's time she was in a profuse sweat, and felt easy, but was indisposed to sleep. The remaining $3^{\text {ss }}$ of laudanum was then given, and towards moming she had some sleep.

First day.-In the morning she expressed herself as feeling comfortable, and free from pain. She breathes easily, and the skin is freely perspiring. Pulse 130 ; tongue moist, slightly furred. The upper part of the abdomen, above the 
bandage, is distended with flatus, so as to bulge up above the edge of the bandage. $\xi \mathrm{vj}$ of dark-coloured urine were drawn off by the catheter. The pupils are contracted, evidently from the effects of opium, and she is in a quiet dozy state, readily, however, answering questions, and quite free from restlessness.

Great care was taken to keep her undisturbed, and she was ordered cold drinks of spring- or toast-water, or tea. In the course of the day she was ordered-Julepium Ammon. Acet. c. Mist. Camph. et Tinct. Hyoscyam. 3ss, $4^{\text {tis }}$ horis, but of this she took only two doses.

In the evening.- She had passed the day in a perfectly tranquil manner, sleeping a good deal. She had taken freely of the cold liquids from time to time, and there was but little alteration in her general state, which was as favorable as could be expected. Between six and seven ounces of clear urine were drawn off. She had sweated profusely at intervals during the day, requiring the clothes to be lightened, and now the skin is warm and moist. There is no abdominal tenderness.

The vulva was sponged with warm water; warm napkins were applied, and the sheets were shifted from under her with as little movement as possible. There was a moderate dark-coloured lochial discharge.-Calomel, gr. j, Pulv. Opii, gr. ij, in formâ Pilul. Statim sumenda.

Another pill was ordered to be given, at the discretion of the resident obstetric clerk who sat up with her, if the first should fail to procure rest.

Second day.-The second pill was given at 2 a.m., and she passed a tolerably quiet night. Towards morning she complained of a sharp shooting pain below the edge of the false ribs on the right side, with short, free intermissions. Pain also was felt in the uterine region, and the abdomen was more tympanitic. She had passed urine of her own accord during the night. Pulse 150, small; respiration more rapid; mind clear. The pain in the side increased towards noon, and the breathing was more embarrassed.

Some cloths wrung out of hot water were applied to the 
side. Another two grains of opium were given, and the vulva again sponged; she had partaken freely of barley- and toast-water, and tea; on one occasion she had vomited, but without effort. In the afternoon her breathing became catching, and more frequent; her pulse more rapid and feeble, and evidence of vital exhaustion was apparent. She died in the evening.

From the time of her delivery to her death, this patient was watched, without intermission, by Mr. Davies and the resident obstetric clerks.

Post-mortem examination.-Thirty-six hours after death an inspection of the abdomen was made by myself with Messrs. Stocker and Morgan, the obstetric assistants. The upper region of the abdomen, above the bandages, was distended by flatus; but the middle and lower parts, which had been covered by plaster, were comparatively flat. On removing the bandage and plaster, the edges of the external wound were seen closely approximated; and on cutting through the sutures, the process of adhesion was found to have made some advance, so that it needed some little traction, beyond the weight of the neighbouring tissues, to reopen the incision. On exposing the abdominal cavity, a few coils of the small intestines, near the uterus, were seen not much injected, but with some films of recent lymph between them, and some serous fluid tinged with blood, and intermixed with flakes of lymph. But the degree of peritonitis was not great, and it did not extend to the upper part of the abdomen. The bowels were distended with gas, but their solid contents were trifling. The omentum was much thickened and indurated, and its blood-vessels were filled with blood. It sunk upon the fundus uteri, and there, being comparatively fixed, it formed a barrier, which prevented the blood from the open uterus running into the peritoneal cavity. The uterus occupied its normal position after delivery ; rather inclined to the left, and well-reduced in size. The length of the incision was reduced to three inches, and its curved outline more distinct than when made during the operation. 
The edges, for almost the entire length, were separated, leaving a gap of a half to three fourths of an inch in width, when measured from the outer surface. The edges, towards the inner surface of the uterus, were nearer together, but still apart, and some lochial discharge ran out through the opening, which, during life, had apparently been retained within the limits of the uterus by the close fitting in of the abdominal walls with the pressure of lint and strapping, and the upper wall of indurated omentum. The length of the uterus was nine inches and a quarter, and if a line were drawn half an inch transversely above the centre, the incision would be contained in the lower part. The peritoneal surface of the uterus was more opaque than natural, with here and there some shreds of recent lymph; but there was but little vascular injection. The muscular structure of the uterus, and the veins were not inflamed or thickened, excepting at the margin of the opening, where the tissue felt more dense and crisper than usual. The os uteri was uninjured. A corpus luteum was in the right ovary. The pelvis, which I was fortunate enough to procure, is altogether small, all its diameters being below the standard measurement, excepting at the outlet, which is sufficiently spacious. The principal deformity is in the conjugate diameter of the brim, on account of the promontory of the sacrum jutting forward with an inclination to the right side. It measures two inches. From the promontory to the right pectineal eminence there is a space of two inches, but to the left pectineal eminence, there is two inches and an eighth. The transverse measurement is four inches and three fourths, and the oblique, four inches. The diameters of the cavity, although contracted, are not so reduced as to cause any serious obstacle, in a practical point of view, to the removal of a child.

Remarks. - There were two practical questions suggested by this case :- 1 . What was the best plan to attempt to follow out in the delivery at first? 2. Were the complieations such as, under the circumstances, to justify the Cæsarean section?

xxxIv. 
1. When first I saw this young woman, she was already seven months advanced in pregnancy, and my conviction, after a vaginal examination, was that there were, as nearly as possible, two inches in the conjugate diameter, which was the particular solid obstruction to be considered in the delivery.

The two methods of treatment which might be adopted, were-(1) to induce premature labour, and deliver by craniotomy, and (2) to allow her to go to term, and then perform the Cæsarean section. By inducing premature labour, although at the age of viability, there was no expectation of saving the child, but only that, by removing a body of relatively small dimensions, the operation by the crotchet would be facilitated. The important favorable circumstance in this mode of delivery was, that it afforded, in my judgment, a reasonable expectation of being completed through the birth-passages by an operation, from which the mother might escape with impunity, although at the sacrifice of the child. In favour of the Cæsarean section, there was the probable preservation of the child, and the somewhat diminished risk from so formidable an operation, by a careful preparation of the patient for it-undertaking it at a selected moment, and such attention to points of practice, during and after it, as have lately been found of service in abdominal surgery. This mode of delivery would, I believe, have found favour in the eyes of some obstetric practitioners, with whom the expectation of saving the child's life would have outweighed the degree of operative danger to which the mother would be exposed. My own judgment was clearly in concurrence with the practice which was adopted. Had the pelvic deformity been greater, and pregnancy, as in this case, been concealed until the seventh month, preventing, therefore, an early induction of labour, there could have been no hesitation in having recourse to the Cæsarean section. But with a conjugate diameter of two inches, and a sufficiently spacious cavity and outlet, there was so fair a likelihood of delivering by the crotchet, that I should have felt culpable in not undertaking it. In the election between the safety of 
the child, and an augmented danger to the mother from a serious surgical operation, I was not disposed, in this case, to tamper with the maxim which happily prevails with obstetric practitioners in England in favour of the mother. And although the results of this case, when separated from these and after-considerations, and appearing nakedly as in a statistical table, are most gloomy and disappointing, and delivery by the Cæsarean section, at first, might have rescued the child, and offered a better chance for the mother, yet I should not, in a similar case, feel justified in deviating from the same practice. My conviction is, that the mal-position of the child, which was, of course, unexpected, and beyond control, so augmented the difficulties of the first hinderance, as to thwart my purpose.

With reference to the second question, it will be noticed, that twelve hours were suffered to elapse to allow the first stage of labour to be well completed, and to favour the physiological softening of the vagina and external organs. In introducing my hand, with the view of learning more accurately the degree of deformity of the pelvis, I had the remote expectation that $I$ might probably seize, with the fingers, a lower extremity, by the same kind of manipulation which Dr. Lee has practised successfully in some cases of unavoidable hæmorrhage, with a rigid undilated os uteri. Failing in this, the prolapsed arm was, with the aid of external pressure, made the means, by traction on it, of bringing the head over the brim, which allowed of its being opened, reduced, and drawn upon, until I hoped to get it through the brim. It was in a pause of this operation that, on sweeping the inlet of the pelvis with the finger, I found that a foot had descended by the side of the hand, and had been squeezed into the right side of the pelvis by the action of the uterus. On watching the effect of some labour-pains, I found that the force of the contractions was spent, not in pressing one part below the other, but in wedging them altogether in the narrow brim. It was obvious that, if either extremity could be dislodged from the brim and brought down, it might be made of great service in helping 
delivery; but all attempts to accomplish this manœuvre failed, as no sufficient hold could be got of them. With this new source of difficulty, what was best to be done? It appeared to me that one of two courses might be followed. The first was to persevere in removing the mutilated fœtus through the vagina; and the second, to deliver by the Cæsarian section. I felt, too, that it was imperative to decide between them at once, as the effect of every half-hour's delay, especially if employed in further efforts at extraction, would increase the danger of the Crsarean section, and, indeed, if long persevered in, would compromise all chance of its success. The patient at this time manifested no signs of exhaustion, the pulse, though rapid, was of good volume; her spirits were fairly good; the abdomen was free from tenderness, and the sexual passages were not inflamed. The principal reason which induced me to prefer the Cæsarean section was, that I feared the patient would not susiain such prolonged efforts at delivery as would have been necessary, and that she might die exhausted before or soon after delivery. The girl had but a feeble constitution, although her health had not, of late years, been bad; and I had no confidence in its enduring against this hard trial of its powers. From the efforts I had already made, and from some experience in severe cases of craniotomy, I was quite sure that it would be a tedious and most difficult task, although aided by a fast advancing putrefaction of the fotus, to effect delivery ; and that even the life of a strong woman would have been placed in peril by it. I could not but call to mind a case which occurred in the Lying-in Charity of Guy's, in October, 1835, at which I was present. The patient, aged 32, was in labour with her sixth child, and the right hand and arm descended at the commencement of labour. An effort was made to turn by the student in attendance, but ineffectually. She was bled to $\bar{z} \mathrm{xvj}$, and took 100 drops of laudanum, when another attempt at version was made by the assistant-accoucheur, which also failed. The pelvis was found to be contracted in all its diameters, and the conjugate diameter measured two inches ten lines. 
Eight hours from the first attempt at delivery, she was removed from her lodgings to a ward prepared for her in the hospital, and Dr. Ashwell then took charge of the case. She had already had a rigor; the pulse was rapid, and the abdomen tender. Without trying again to turn, Dr. Ashwell made attempts to remove the child by the cutting hook; but it could not be fixed upon the neck to sever the head from the trunk. The thorax was perforated; but after the most strenuous efforts, perseveringly followed out for many hours, it was obliged to be abandoned as hopeless, and the woman died undelivered. The pelvis is now upon the table. "If this case could again occur," says Dr. Ashwell, (vide 'Guy's Hospital Reports,' Vol. I,) " at the full period, and the knowledge now attained were possessed, there would, I think, be little hesitation as to the propriety of the Cæsarean section."

Another unfavorable circumstance in this case, in reference to persisting with the crotchet, was the state of the vagina. This canal has been noticed as retaining the structure of early age. It was closely ribbed throughout, very vascular, with a thin epithelial covering, and a feeling imparted to the finger of its being structurally weak and easily lacerable. This was remarked to those around me at the beginning, as a source of danger in a prolonged craniotomy case; and, excepting when the hand was introduced which lacerated the fourchette, this canal was very carefully guarded when the crotchet was being used. Should the patient survive the immediate dangers of delivery, I could not help feeling that there was a great risk of vaginal sloughing, with its revolting consequences.

On the other hand, the Cæsarean section offered a speedy and sure, instead of a prolonged and doubtful delivery, it was not yet forbidden from exhaustion or any signs of inflammation; on the score of suffering, it contrasted most favorably with the other practice, and I thought, upon the whole, that the chance of ultimate success from it was greater.

There is but little need of comment on the operation 
itself, beyond the detail which has been given of it. It was performed much in the usual way without difficulty, and with nothing untoward. The incision was made as low down in the front wall as possible, and the slight curve in its direction was suggested by me, with the hope of catching: the outline of the muscular fibres of the inner surface of the uterus, and so far favouring the closure of the wound within. It certainly failed to do so, and I do not know that there was any advantage over the straight incision.

The little hæmorrhage which attended the operation, and which did not delay the closure of the external wound, was, in great measure, due, I conclude, to the uterus being at the seventh month of gestation instead of the ninth, to the placenta being attached to the posterior wall, and to the incision being low down in the uterus, where the veins would be less developed.

The influence of the chloroform appeared to me to be a gain in every way.

It will be seen that the after-treatment by opium was steadily followed out, and the repetition of the doses was only suspended when the patient appeared at the time to be under its influence. The extent of reparation of the external wound, and the admirable manner in which the structures were laid out, to limit the mischief from the open wound in the uterus, and prevent its extravasated contents being diffused in the abdominal cavity, are worthy of attention, and must have been greatly favoured by the perfect tranquillity of the patient under the influence of opium. 